\title{
Spin-orbit-coupled Bose-Einstein condensates
}

\author{
Y.-J. Lin ${ }^{1}$, K. Jiménez-García ${ }^{1,2} \&$ I. B. Spielman ${ }^{1}$ \\ ${ }^{1}$ Joint Quantum Institute, National Institute of Standards and Technology, and University of Mary- \\ land, Gaithersburg, Maryland, 20899, USA \\ ${ }^{2}$ Departamento de Física, Centro de Investigación y Estudios Avanzados del Instituto Politécnico \\ Nacional, México D.F., 07360, México
}

Spin-orbit (SO) coupling - the interaction between a quantum particle's spin and its momentum - is ubiquitous in nature, from atoms to solids. In condensed matter systems, SO coupling is crucial for the spin-Hall effect ${ }^{1 / 2}$ and topological insulators ${ }^{3+5}$, which are of extensive interest; it contributes to the electronic properties of materials such as GaAs, and is important for spintronic devices ${ }^{6}$. Ultracold atoms, quantum many-body systems under precise experimental control, would seem to be an ideal platform to study these fascinating SO coupled systems. While an atom's intrinsic SO coupling affects its electronic structure, it does not lead to coupling between the spin and the center-of-mass motion of the atom. Here, we engineer SO coupling (with equal Rashba ${ }^{7}$ and Dresselhaus $^{8}$ strengths) in a neutral $^{8}$ atomic Bose-Einstein condensate by dressing two atomic spin states with a pair of lasers ${ }^{\text {? }}$. Not only is this the first SO coupling realized in ultracold atomic gases, it is also the first ever for bosons. Furthermore, in the presence of the laser coupling, the interactions between the two dressed atomic spin states are modified, driving a quantum phase transition from a spatially spin-mixed state (lasers off) to a phase separated state (above a critical laser intensity). 


\section{The location of this transition is in quantitative agreement with our theory. This SO coupling}

- equally applicable for bosons and fermions - sets the stage to realize topological insulators in fermionic neutral atom systems.

Quantum particles have an internal "spin" angular momentum; this can be intrinsic for fundamental particles like electrons, or a combination of intrinsic (from nucleons and electrons) and orbital for composite particles like atoms. Spin-Orbit (SO) coupling links a particle's spin to its motion, and generally appears for particles moving in static electric fields, such as the nuclear field of an atom or the crystal field in a material. The coupling results from the Zeeman interaction $-\vec{\mu} \cdot \vec{B}$ between a particle's magnetic moment $\vec{\mu}$, parallel to the spin $\vec{\sigma}$, and a magnetic field $\vec{B}$ present in the frame moving with the particle. For example, Maxwell's equations dictate that a static electric field $\vec{E}=E_{0} \hat{z}$ in the lab frame (at rest) gives a magnetic field $\vec{B}_{\mathrm{SO}}=E_{0}\left(\hbar / m c^{2}\right)\left(-k_{y}, k_{x}, 0\right)$ in the frame of an object moving with momentum $\hbar \vec{k}=\hbar\left(k_{x}, k_{y}, k_{z}\right)$, where $c$ is the speed of light in vacuum and $m$ is the particle's mass. The resulting momentum dependent Zeeman interaction $-\vec{\mu} \cdot \vec{B}_{\mathrm{SO}}(\mathbf{k}) \propto \sigma_{x} k_{y}-\sigma_{y} k_{x}$ is known as the Rashba ${ }^{7} \mathrm{SO}$ coupling. In combination with the Dresselhaus $^{8}$ coupling $\propto-\sigma_{x} k_{y}-\sigma_{y} k_{x}$, these describe two dimensional (2D) SO coupling in solids to first order.

In materials, the SO coupling strengths are generally intrinsic properties, which are largely determined by the specific material and the details of its growth, and thus only slightly adjustable in the laboratory. We demonstrate SO coupling in a ${ }^{87} \mathrm{Rb}$ Bose-Einstein condensate (BEC) where a pair of Raman lasers create a momentum-sensitive coupling between two internal atomic states. This SO coupling is equivalent to that of an electronic system with equal contributions of Rashba 
and Dresselhaus ${ }^{9}$ couplings, and with a uniform magnetic field $\vec{B}$ in the $\hat{y}-\hat{z}$ plane, which is described by the single particle Hamiltonian

$$
\hat{H}=\frac{\hbar^{2} \hat{\mathbf{k}}^{2}}{2 m} \check{1}-\left[\vec{B}+\vec{B}_{\mathrm{SO}}(\hat{\mathbf{k}})\right] \cdot \vec{\mu}=\frac{\hbar^{2} \hat{\mathbf{k}}^{2}}{2 m} \check{1}+\frac{\Omega}{2} \check{\sigma}_{z}+\frac{\delta}{2} \check{\sigma}_{y}+2 \alpha \hat{k}_{x} \check{\sigma}_{y} .
$$

$\alpha$ parametrizes the SO coupling strength; $\Omega=-g \mu_{\mathrm{B}} B_{z}$ and $\delta=-g \mu_{\mathrm{B}} B_{y}$ result from the Zeeman fields along $\hat{z}$ and $\hat{y}$, respectively; and $\check{\sigma}_{x, y, z}$ are the $2 \times 2$ Pauli matrices. Absent SO coupling, electrons have group velocity $v_{x}=\hbar k_{x} / m$, independent of their spin. With SO coupling, their velocity becomes spin-dependent, $v_{x}=\hbar\left(k_{x} \pm 2 \alpha m / \hbar^{2}\right) / m$ for spin $|\uparrow\rangle$ and $|\downarrow\rangle$ electrons (quantized along $\hat{y}$ ). In two recent experiments, this form of SO coupling was engineered in GaAs heterostructures where confinement into 2D planes linearized GaAs's native cubic SO coupling to produce a Dresselhaus term, and asymmetries in the confining potential gave rise to Rashba coupling. In one experiment a persistent spin helix was found ${ }^{6}$, and in another the SO coupling was only revealed by adding a Zeeman field ${ }^{10}$.

SO coupling for neutral atoms enables a range of exciting experiments, and importantly, it is a key ingredient to realize neutral atom topological insulators. Topological insulators are novel fermionic band insulators including integer-, and now spin-quantum Hall states that insulate in the bulk, but conduct in topologically protected quantized edge channels. The first known topological insulators - integer quantum Hall states ${ }^{11}$ - require large magnetic fields that explicitly break timereversal symmetry. In a seminal paper ${ }^{3}$, Kane and Mele showed that in some cases SO coupling leads to zero magnetic field topological insulators preserving time-reversal symmetry. Absent the bulk conductance that plagues current materials, cold atoms can potentially realize these insulators in their most pristine form, perhaps revealing their quantized edge (in 2D) or surface (in 3D) states. 
To go beyond the form of SO coupling we created, virtually any SO coupling, including that needed for topological insulators, is possible with additional lasers $\frac{12}{14}$.

To create SO coupling, we select two internal "spin" states from within the ${ }^{87} \mathrm{Rb} 5 \mathrm{~S}_{1 / 2}, F=1$ ground electronic manifold, and label them pseudo-spin up and down in analogy with an electron's two spin states: $|\uparrow\rangle=\left|F=1, m_{F}=0\right\rangle$ and $|\downarrow\rangle=\left|F=1, m_{F}=-1\right\rangle$. A pair of $\lambda=804.1 \mathrm{~nm}$ Raman lasers, intersecting at $\theta=90^{\circ}$ and detuned by $\delta$ from Raman resonance (Fig. 17), couple these states with strength $\Omega$; here $\hbar k_{L}=\sqrt{2} \pi \hbar / \lambda$ and $E_{L}=\hbar^{2} k_{L}^{2} / 2 m$ are the natural units of momentum and energy. In this configuration, the atomic Hamiltonian is given by Eq. 1, with $k_{x}$ replaced by a quasimomentum $q$ and an overall $E_{L}$ energy offset. $\Omega$ and $\delta$ give rise to effective Zeeman fields along $\hat{z}$ and $\hat{y}$, respectively. The SO coupling term $2 E_{L} q \check{\sigma}_{y} / k_{L}$ results from the laser geometry, and $\alpha=E_{L} / k_{L}$ is set by $\lambda$ and $\theta$, independent of $\Omega$ (see Methods). In contrast with the electronic case, the atomic Hamiltonian couples bare atomic states $\left|\uparrow, \kappa_{x}=q+k_{L}\right\rangle$ and $\left|\downarrow, \kappa_{x}=q-k_{L}\right\rangle$ with different velocities, $\hbar \kappa_{x} / m=\hbar\left(q \pm k_{L}\right) / m$.

The spectrum, a new energy-quasimomentum dispersion of the SO coupled Hamiltonian, is displayed in Fig. $1 \mathrm{p}$ at $\delta=0$ and for a range of couplings $\Omega$. The dispersion is divided into upper and lower branches $E_{ \pm}(q)$, and we focus on $E_{-}(q)$. For $\Omega<4 E_{L}$ and small $\delta$ (see Fig. 2a), $E_{-}(q)$ consists of a double-well in quasi-momentum ${ }^{15}$, where the group velocity $\partial E_{-}(q) / \partial \hbar q$ is zero. States near the two minima are dressed spin states, labeled as $\left|\uparrow^{\prime}\right\rangle$ and $\left|\downarrow^{\prime}\right\rangle$. As $\Omega$ increases, the two dressed spin states merge into a single minimum and the simple picture of two dressed spins is inapplicable. Instead, that strong coupling limit effectively describes spinless bosons with a tunable dispersion relation ${ }^{16}$ with which we engineered synthetic electric ${ }^{17}$ and magnetic fields ${ }^{18}$ 
for neutral atoms.

Absent Raman coupling, atoms with spins $|\uparrow\rangle$ and $|\downarrow\rangle$ spatially mixed perfectly in a BEC. By increasing $\Omega$ we observed an abrupt quantum phase transition to a new state where the two dressed spins spatially separated, resulting from a modified effective interaction between the dressed spins.

We studied SO coupling in oblate ${ }^{87} \mathrm{Rb}$ BECs with $\approx 1.8 \times 10^{5}$ atoms in a $\lambda=1064 \mathrm{~nm}$ crossed dipole trap with frequencies $\left(f_{x}, f_{y}, f_{z}\right) \approx(50,50,140) \mathrm{Hz}$. The bias magnetic field $B_{0} \hat{y}$ generated $\mathrm{a} \omega_{Z} / 2 \pi \approx 4.81 \mathrm{MHz}$ Zeeman shift between $|\uparrow\rangle$ and $|\downarrow\rangle$. The Raman beams propagated along $\hat{y} \pm \hat{x}$ and had a constant frequency difference $\Delta \omega_{L} / 2 \pi \approx 4.81 \mathrm{MHz}$. The small detuning from Raman resonance $\delta=\hbar\left(\Delta \omega_{L}-\omega_{Z}\right)$ was set by $B_{0}$, and $\left|m_{F}=+1\right\rangle$ was decoupled due to the quadratic Zeeman effect (see Methods).

We prepared BECs with an equal population of $|\uparrow\rangle$ and $|\downarrow\rangle$ at $\Omega, \delta=0$, adiabatically increased $\Omega$ to a final value up to $7 E_{L}$ in $70 \mathrm{~ms}$, and then allowed the system to equilibrate for $t_{h}=70 \mathrm{~ms}$. We abruptly ( $t_{\mathrm{off}}<1 \mu \mathrm{s}$ ) turned off the Raman lasers and the dipole trap-thus projecting the dressed state onto their constituent bare spin and momentum states-and absorption-imaged them after a $30.1 \mathrm{~ms}$ time-of-flight (TOF). For $\Omega>4 E_{L}$ (Fig. 1 d ), the BEC was located at the single minimum $q_{0}$ of $E_{-}(q)$ with a single momentum component in each spin state corresponding to the pair $\left\{\left|\uparrow, q_{0}+k_{L}\right\rangle,\left|\downarrow, q_{0}-k_{L}\right\rangle\right\}$. However, for $\Omega<4 E_{L}$ we observed two momentum components in each spin state, corresponding to the two minima of $E_{-}(q)$ at $q_{\uparrow}$ and $q_{\downarrow}$. The agreement between the data (symbols), and the expected minima-locations (curves), demonstrates the existence of the SO coupling associated with the Raman dressing. We maintained $\delta \approx 0$ when turning 
on $\Omega$ by making equal populations in bare spins $|\uparrow\rangle,|\downarrow\rangle$ (see Fig. 1 $1 \mathrm{~d}$ ).

We experimentally studied the low temperature phases of these interacting SO coupled bosons as a function of $\Omega$ and $\delta$. The zero-temperature mean-field phase diagram (Fig. $2 \mathrm{a}, \mathrm{b}$ ) includes phases composed of: a single dressed spin state, a spatial mixture of both dressed spin states, and coexisting but spatially phase-separated dressed spins.

This phase diagram can be largely understood from non-interacting bosons condensing into the lowest energy single particle state, and can be divided into three regimes (Fig. 2a). In the region of positive detuning marked $\left|\downarrow^{\prime}\right\rangle$, there are double minima at $q=q_{\uparrow}, q_{\downarrow}$ in $E_{-}(q)$ with $E_{-}\left(q_{\downarrow}\right)<E_{-}\left(q_{\uparrow}\right)$ and the bosons condense at $q_{\downarrow}$. In the region marked $\left|\uparrow^{\prime}\right\rangle$ the reverse holds. The energy difference between the two minima is $\Delta(\Omega, \delta)=E_{-}\left(q_{\uparrow}\right)-E_{-}\left(q_{\downarrow}\right) \approx \delta$ for small $\delta$ (see Methods). In the third "single minimum" regime, the atoms condense at the single minimum $q_{0}$. These dressed spins act as free particles with group velocity $\hbar K_{x} / m$ (with an effective mass $m^{*} \approx m$, for small $\Omega$ ), where $K_{x}=q-q_{\uparrow, \downarrow, 0}$ for the different minima.

We investigated the phase diagram using BECs with initially equal spin populations prepared as described previously, but with $\delta \neq 0$ and $t_{h}$ up to $3 \mathrm{~s}$. We probed the atoms after abruptly removing the dipole trap, and then ramping $\Omega \rightarrow 0$ in $1.5 \mathrm{~ms}$. This approximately mapped $\left|\uparrow^{\prime}\right\rangle$ and $\left|\downarrow^{\prime}\right\rangle$ back to their undressed counterparts $|\uparrow\rangle$ and $|\downarrow\rangle$ (see Methods). We absorption-imaged the atoms after a $30 \mathrm{~ms}$ TOF, during the last $20 \mathrm{~ms}$ of which a Stern-Gerlach magnetic field gradient along $\hat{y}$ separated the spin components.

Figure 3 a shows the condensate fraction $f_{\downarrow^{\prime}}=N_{\downarrow^{\prime}} /\left(N_{\downarrow^{\prime}}+N_{\uparrow^{\prime}}\right)$ in $\left|\downarrow^{\prime}\right\rangle$ at $\Omega=0.6 E_{L}$ as a 
function of $\delta$, at $t_{h}=0.1,1$ and $3 \mathrm{~s}$, where $N_{\uparrow^{\prime}}$ and $N_{\downarrow^{\prime}}$ denote the number of condensed atoms in $\left|\uparrow^{\prime}\right\rangle$ and $\left|\downarrow^{\prime}\right\rangle$, respectively. The BEC is all $\left|\uparrow^{\prime}\right\rangle$ for $\delta \lesssim 0$ and all $\left|\downarrow^{\prime}\right\rangle$ for $\delta \gtrsim 0$, but both dressed spin populations substantially coexisted for detunings within $\pm w_{\delta}$ (obtained by fitting $f_{\downarrow^{\prime}}$ to the error function where $\delta= \pm w_{\delta}$ corresponds to $f_{\downarrow^{\prime}}=0.50 \pm 0.16$ ). Figure $3 \mathrm{p}$ shows $w_{\delta}$ versus $\Omega$ for hold times $t_{h} . w_{\delta}$ decreases with $t_{h}$; even by our longest $t_{h}=3 \mathrm{~s}$ it has not reached equilibrium.

Conventional $F=1$ spinor BECs have been studied in ${ }^{23} \mathrm{Na}$ and ${ }^{87} \mathrm{Rb}$ without Raman coupling $19-21$. For our $|\uparrow\rangle$ and $|\downarrow\rangle$ states, the interaction energy depends on the local density in each spin state, and is described by

$$
\hat{H}_{\mathrm{I}}=\frac{1}{2} \int d^{3} r\left[\left(c_{0}+\frac{c_{2}}{2}\right)\left(\hat{\rho}_{\uparrow}+\hat{\rho}_{\downarrow}\right)^{2}+\frac{c_{2}}{2}\left(\hat{\rho}_{\downarrow}^{2}-\hat{\rho}_{\uparrow}^{2}\right)+\left(c_{2}+c_{\uparrow \downarrow}^{\prime}\right) \hat{\rho}_{\uparrow} \hat{\rho}_{\downarrow}\right]
$$

where $\hat{\rho}_{\uparrow}$ and $\hat{\rho}_{\downarrow}$ are density operators for $|\uparrow\rangle$ and $|\downarrow\rangle$. In the ${ }^{87} \mathrm{Rb} F=1$ manifold, the spin independent interaction is $c_{0}=7.79 \times 10^{-12} \mathrm{~Hz} \mathrm{~cm}^{3}$, the spin dependent interaction ${ }^{22}$ is $c_{2}=$ $-3.61 \times 10^{-14} \mathrm{~Hz} \mathrm{~cm}^{3}$, and $c_{\uparrow \downarrow}^{\prime}=0$. Since $\left|c_{0}\right| \gg\left|c_{2}\right|$ the interaction is almost spin independent, but because $c_{2}<0$, the two-component mixture of $|\uparrow\rangle$ and $|\downarrow\rangle$ has a spatially mixed ground state (is miscible). When $\hat{H}_{\mathrm{I}}$ is re-expressed in terms of the dressed spin states, $c_{\uparrow \downarrow}^{\prime} \approx c_{0} \Omega^{2} /\left(8 E_{L}^{2}\right)$ is nonzero and corresponds to an effective interaction between $\left|\uparrow^{\prime}\right\rangle$ and $\left|\downarrow^{\prime}\right\rangle$. This modifies the ground state of our SO coupled BEC (mixtures of $\left|\uparrow^{\prime}\right\rangle$ and $\left|\downarrow^{\prime}\right\rangle$ ) from phase-mixed to phase-separated above a critical Raman coupling strength $\Omega_{c}$. This transition lies outside the common single-mode approximation 20 .

The effective interaction between $\left|\uparrow^{\prime}\right\rangle$ and $\left|\downarrow^{\prime}\right\rangle$ is an exchange energy resulting from the non-orthogonal spin part of $\left|\uparrow^{\prime}\right\rangle$ and $\left|\downarrow^{\prime}\right\rangle$ (see Methods): a spatial mixture produces total den- 
sity modulations ${ }^{15}$ with wavevector $2 k_{L}$ in analogy with the spin-textures of the electronic case ${ }^{6}$. These increase the state-independent interaction energy in $\hat{H}_{\mathrm{I}}$ wherever the two dressed spins spatially overlap, contributing to the $c_{\uparrow \downarrow}^{\prime}$ term. (Such a term does not appear for rf-dressed states, which are always spin-orthogonal.) Because $c_{\uparrow \downarrow}^{\prime}$ and $c_{2}$ have opposite sign here, the dressed BEC can go from miscible to immiscible, at the miscibility threshold ${ }^{19}$ for a two-component BEC $c_{0}+c_{2}+c_{\uparrow \downarrow}^{\prime} / 2=\sqrt{c_{0}\left(c_{0}+c_{2}\right)}$, when $\Omega=\Omega_{c}$ (this result is in agreement with an independent theory presented in Ref. ${ }^{23}$ ).

Figure $2 \mathrm{~b}$ depicts the mean field phase diagram including interactions, computed by minimizing the interaction energy $H_{\mathrm{I}}$ plus the single particle detuning $\Delta(\Omega, \delta) \approx \delta$. This phase diagram adds to the non-interacting picture both mixed (hashed) and phase-separated (bold line) regimes. The $c_{2}\left(\hat{\rho}_{\downarrow}^{2}-\hat{\rho}_{\uparrow}^{2}\right) / 2$ term in $\hat{H}_{\mathrm{I}}$ implies that the energy difference between a $|\uparrow\rangle$ BEC and a $|\downarrow\rangle$ BEC is proportional $N^{2} c_{2}$. The detuning required to compensate for this difference slightly displaces the symmetry point of the phase diagram downwards. As evidenced by the width of the metastable window $2 w_{\delta}$ in Fig. 2 , for $|\delta|<w_{\delta}$ the spin-population does not have time to relax to equilibrium. Since the miscibility condition does not depend on atom number, the phase line in Fig. $2 \mathrm{r}$ shows the system's phases for $|\delta|<w_{\delta}$ : phase-mixed for $\Omega<\Omega_{c}$ and phase-separated for $\Omega>\Omega_{c}$ where $\Omega_{c} \approx \sqrt{-8 c_{2} / c_{0}} E_{L} \approx 0.19 E_{L}$.

We measured the miscibility of the dressed spin components from their spatial profiles after TOF, for $\Omega=0$ to $2 E_{L}$ and $\delta \approx 0$ such that $N_{\mathrm{T} \uparrow^{\prime}} \approx N_{\mathrm{T} \downarrow^{\prime}}$, where $N_{\mathrm{T} \uparrow^{\prime}, \downarrow^{\prime}}$ is the total atom number including both the condensed and thermal components in $\left|\uparrow^{\prime}\right\rangle,\left|\downarrow^{\prime}\right\rangle$. For each TOF image, we numerically recentered the Stern-Gerlach-separated spin distributions (Fig. $2 \mathrm{k}$, and see Methods), 
giving condensate densities $n_{\uparrow^{\prime}}(x, y)$ and $n_{\downarrow^{\prime}}(x, y)$. Since the self-similar expansion of BECs released from harmonic traps essentially magnifies the in-situ spatial spin distribution, these reflect the in-situ densities ${ }^{24}$.

A dimensionless metric $s=1-\left\langle n_{\uparrow^{\prime}} n_{\downarrow^{\prime}}\right\rangle /\left(\left\langle n_{\uparrow^{\prime}}^{2}\right\rangle\left\langle n_{\downarrow^{\prime}}^{2}\right\rangle\right)^{1 / 2}$ quantifies the degree of phase separation $(\langle\ldots\rangle$ is the spatial average over a single image): $s=0$ for any perfect mixture $n_{\uparrow^{\prime}}(x, y) \propto n_{\downarrow^{\prime}}(x, y)$, and $s=1$ for complete phase separation. Figure 4 displays $s$ versus Raman coupling $\Omega$ with a hold time $t_{h}=3 \mathrm{~s}$, showing that $s \approx 0$ for small $\Omega$ (as expected given our miscible bare spins) and $s$ abruptly increases above a critical $\Omega_{c}$. The inset to Fig. 4 plots $s$ as a function of time, showing that $s$ reaches steady-state in $0.14(3) \mathrm{s} \ll t_{h}$. To obtain $\Omega_{c}$, we fit the data in Fig. 4 to a slowly increasing function below $\Omega_{c}$ and the power-law $1-\left(\Omega / \Omega_{c}\right)^{-a}$ above $\Omega_{c}$. The resulting $\Omega_{c}=0.20(2) E_{L}$ is in agreement with the mean field prediction $\Omega_{c}=0.19 E_{L}$. This demonstrates a quantum phase transition for a two-component SO coupled BEC, from miscible when $\Omega<\Omega_{c}$ to immiscible when $\Omega>\Omega_{c}$.

Even below $\Omega_{c}, s$ slowly increased with increasing $\Omega$. To understand this effect, we numerically solved the 2D spinor Gross-Pitaevskii equation in the presence of a trapping potential. This demonstrated that the differential interaction term $c_{2}\left(\hat{\rho}_{\downarrow}^{2}-\hat{\rho}_{\uparrow}^{2}\right) / 2$ in $\hat{H}_{I}$ favors slightly different density profiles for each spin component, while the $\left(c_{2}+c_{\uparrow \downarrow}^{\prime}\right) \hat{\rho}_{\uparrow} \hat{\rho}_{\downarrow}$ term favors matched profiles. Thus, as $c_{2}+c_{\uparrow \downarrow}^{\prime}$ approached zero from below this balancing effect decreased, leading $s$ to increase.

An infinite system should fully phase separate $(s=1)$ for all $\Omega>\Omega_{c}$. In our finite system, the boundary between the phase separated spins, set by the spin-healing length $\left(\xi_{s}=\right.$ 
$\sqrt{\hbar^{2} / 2 m\left|c_{2}+c_{\uparrow \downarrow}^{\prime}\right| n}$, where $n$ is the local density), can be comparable to the system size. We interpret the increase of $s$ above $\Omega_{c}$ as resulting from the decrease of $\xi_{s}$ with increasing $\Omega$.

We realized SO coupling in a ${ }^{87} \mathrm{Rb} \mathrm{BEC}$, and observed a quantum phase transition from spatially mixed to spatially separated. By operating at lower magnetic field (with a smaller quadratic Zeeman shift), our method extends to the full $F=1$ or $F=2$ manifold of ${ }^{87} \mathrm{Rb}$ or ${ }^{23} \mathrm{Na}$, enabling a new kind of tuning for spinor BECs, without the losses associated with Feshbach tuning 25 . Such modifications may allow access to the expected non-abelian vortices in some $F=2$ condensates $^{26}$. Since our SO coupling is in the small $\Omega$ limit, this technique is practical for fermionic ${ }^{40} \mathrm{~K}$, with its smaller fine-structure splitting and thus larger spontaneous emission rate ${ }^{27}$. When the Fermi energy lies in the gap between the lower and upper bands (e.g., Fig. 1b) there will be a single Fermi surface; this situation can induce $p$-wave coupling between fermions ${ }^{28}$ and more recent work anticipates the appearance of Majorana fermions $\mathrm{s}^{29}$.

\section{Methods Summary}

System preparation Our experiments began with nearly pure $\approx 1.8 \times 10^{5}$ atom ${ }^{87} \mathrm{Rb}$ BECs in the $\left|F=1, m_{F}=-1\right\rangle$ state $^{30}$ confined in a crossed optical dipole trap. The trap consisted of a pair of $1064 \mathrm{~nm}$ laser beams propagating along $\hat{x}-\hat{y}\left(1 / e^{2}\right.$ radii of $w_{\hat{x}+\hat{y}} \approx 120 \mu \mathrm{m}$ and $\left.w_{\hat{z}} \approx 50 \mu \mathrm{m}\right)$ and $-\hat{x}-\hat{y}\left(1 / e^{2}\right.$ radii of $\left.w_{\hat{x}-\hat{y}} \approx w_{\hat{z}} \approx 65 \mu \mathrm{m}\right)$.

We prepared equal mixtures of $\left|F=1, m_{F}=-1\right\rangle$ and $|1,0\rangle$ using an initially off resonant rf magnetic field $B_{\mathrm{rf}}(t) \hat{x}$. We adiabatically ramped $\delta$ to $\delta \approx 0$ in $15 \mathrm{~ms}$, decreased the rf coupling 
strength $\Omega_{\mathrm{rf}}$ to about $150 \mathrm{~Hz} \ll \hbar \omega_{q}$ in $6 \mathrm{~ms}$, and suddenly turned off $\Omega_{\mathrm{rf}}$, projecting the BEC into an equal superposition of $\left|m_{F}=-1\right\rangle$ and $\left|m_{F}=0\right\rangle$. We subsequently ramped $\delta$ to its desired value in $6 \mathrm{~ms}$ and then linearly increased the intensity of the Raman lasers from zero to the final coupling $\Omega$ in $70 \mathrm{~ms}$.

Magnetic fields Three pairs of Helmholtz coils, orthogonally aligned along $\hat{x}+\hat{y}, \hat{x}-\hat{y}$ and $\hat{z}$, provided bias fields $\left(B_{x+y}, B_{x-y}\right.$, and $\left.B_{z}\right)$. By monitoring the $\left|F=1, m_{F}=-1\right\rangle$ and $|1,0\rangle$ populations in a nominally resonant rf dressed state, prepared as above, we observed a short-time (below $\approx 10$ minutes) RMS field stability $g \mu_{\mathrm{B}} B_{\mathrm{RMS}} / h \lesssim 80 \mathrm{~Hz}$. The field drifted slowly on longer time scales (but changed abruptly when unwary colleagues entered through our laboratory's ferromagnetic doors). We compensated for the drift by tracking the rf and Raman resonance conditions.

Due to the small energy scales involved in the experiment, it was crucial to minimize magnetic field gradients. We detected stray gradients by monitoring the spatial distribution of $\left|m_{F}=-1\right\rangle$ $\left|m_{F}=0\right\rangle$ spin mixtures after TOF. Small magnetic field gradients caused this otherwise miscible mixture to phase separate along the direction of the gradient. We canceled the gradients in the $\hat{x}-\hat{y}$ plane with two pairs of anti-Helmholtz coils, aligned along $\hat{x}+\hat{y}$ and $\hat{x}-\hat{y}$, to $g \mu_{B} B^{\prime} / h \lesssim 0.7 \mathrm{~Hz} / \mu \mathrm{m}$

1. Kato, Y. K., Myers, R. C., Gossard, A. C. \& Awschalom, D. D. Observation of the Spin Hall Effect in Semiconductors. Science 306, 1910-1913 (2004).

2. Konig, M. et al. Quantum Spin Hall Insulator State in HgTe Quantum Wells. Science 318, 766-770 (2007). 
3. Kane, C. L. \& Mele, E. J. $Z_{2}$ topological order and the quantum spin Hall effect. Phys. Rev. Lett. 95, 146802 (2005).

4. Bernevig, B. A., Hughes, T. L. \& Zhang, S.-C. Quantum Spin Hall Effect and Topological Phase Transition in HgTe Quantum Wells. Science 314, 1757-1761 (2006).

5. Hsieh, D. et al. A topological Dirac insulator in a quantum spin Hall phase. Nature 452, 970-974 (2008).

6. Koralek, J. D. et al. Emergence of the persistent spin helix in semiconductor quantum wells. Nature 458, 610-613 (2009).

7. Bychkov, Y. A. \& Rashba, E. I. Oscillatory effects and the magnetic susceptibility of carriers in inversion layers. Journal of Physics C: Solid State Physics 17, 6039 (1984).

8. Dresselhaus, G. Spin-orbit coupling effects in zinc blende structures. Phys. Rev. 100, 580-586 (1955).

9. Liu, X.-J., Borunda, M. F., Liu, X. \& Sinova, J. Effect of induced spin-orbit coupling for atoms via laser fields. Phys. Rev. Lett. 102, 046402 (2009).

10. Quay, C. H. L. et al. Observation of a one-dimensional spin-orbit gap in a quantum wire. Nat Phys 6, 336-339 (2010).

11. von Klitzing, K., Dorda, G. \& Pepper, M. New method for high-accuracy determination of the fine-structure constant based on quantized Hall resistance. Phys. Rev. Lett. 45, 494-497 (1980). 
12. Ruseckas, J., Juzeliūnas, G., Öhberg, P. \& Fleischhauer, M. Non-abelian gauge potentials for ultracold atoms with degenerate dark states. Phys. Rev. Lett. 95, 010404 (2005).

13. Stanescu, T. D., Zhang, C. \& Galitski, V. Nonequilibrium spin dynamics in a trapped Fermi gas with effective spin-orbit interactions. Phys. Rev. Lett. 99, 110403 (2007).

14. Dalibard, J., Gerbier, F., Juzeliūnas, G. \& Öhberg, P. Artificial gauge potentials for neutral atoms. arXiv:1008.5378v1 (2010).

15. Higbie, J. \& Stamper-Kurn, D. M. Generating macroscopic-quantum-superposition states in momentum and internal-state space from bose-einstein condensates with repulsive interactions. Phys. Rev. A 69, 053605 (2004).

16. Lin, Y.-J. et al. Bose-Einstein condensate in a uniform light-induced vector potential. Phys. Rev. Lett. 102, 130401 (2009).

17. Lin, Y.-J. et al. A synthetic electric force acting on neutral atoms. arXiv:1008.4864v1 (2011).

18. Lin, Y. J., Compton, R. L., Jimenez-Garcia, K., Porto, J. V. \& Spielman, I. B. Synthetic magnetic fields for ultracold neutral atoms. Nature 462, 628-632 (2009).

19. Stenger, J. et al. Spin domains in ground-state Bose-Einstein condensates. Nature 396, 345348 (1998).

20. Chang, M.-S. et al. Observation of spinor dynamics in optically trapped ${ }^{87} \mathrm{Rb}$ Bose-Einstein condensates. Phys. Rev. Lett. 92, 140403 (2004).

21. Ho, T.-L. Spinor Bose condensates in optical traps. Phys. Rev. Lett. 81, 742-745 (1998). 
22. Widera, A. et al. Precision measurement of spin-dependent interaction strengths for spin-1 and spin-2 ${ }^{87} \mathrm{Rb}$ atoms. New J. Phys. 8, 152 (2006).

23. Ho, T.-L. \& Zhang, S. Bose-Einstein condensates in non-abelian gauge fields. arXiv:1007.0650 (2010).

24. Hall, D. S., Matthews, M. R., Ensher, J. R., Wieman, C. E. \& Cornell, E. A. Dynamics of component separation in a binary mixture of Bose-Einstein condensates. Phys. Rev. Lett. 81, 1539-1542 (1998).

25. Erhard, M., Schmaljohann, H., Kronjäger, J., Bongs, K. \& Sengstock, K. Measurement of a mixed-spin-channel Feshbach resonance in ${ }^{87}$ Rb. Phys. Rev. A 69, 032705 (2004).

26. Kobayashi, M., Kawaguchi, Y., Nitta, M. \& Ueda, M. Collision dynamics and rung formation of non-abelian vortices. Phys. Rev. Lett. 103, 115301 (2009).

27. Goldman, N. et al. Realistic time-reversal invariant topological insulators with neutral atoms. Phys. Rev. Lett. 105, 255302 (2010).

28. Zhang, C., Tewari, S., Lutchyn, R. M. \& Das Sarma, S. $p_{x}+i p_{y}$ superfluid from $s$-wave interactions of fermionic cold atoms. Phys. Rev. Lett. 101, 160401 (2008).

29. Sau, J. D., Tewari, S., Lutchyn, R. M., Stanescu, T. D. \& Das Sarma, S. Non-abelian quantum order in spin-orbit-coupled semiconductors: Search for topological majorana particles in solidstate systems. Phys. Rev. B 82, 214509 (2010). 
30. Lin, Y.-J., Perry, A. R., Compton, R. L., Spielman, I. B. \& Porto, J. V. Rapid production of ${ }^{87} \mathrm{Rb}$ Bose-Einstein condensates in a combined magnetic and optical potential. Phys. Rev. A 79, 063631 (2009).

Acknowledgements We thank E. Demler, T.-L. Ho, and H. Zhai for conceptual input; and we appreciate conversations with J. V. Porto, and W. D. Phillips. This work was partially supported by ONR, ARO with funds from the DARPA OLE program, and the NSF through the Physics Frontier Center at JQI. K.J.-G. acknowledges CONACYT.

Competing Interests The authors declare that they have no competing financial interests.

Author Contributions All authors contributed to writing of the manuscript. Y.-J. L. lead the data taking effort in which K. J.-G. participated. I. B. S. conceived the experiment; performed numerical and analytic calculations; and supervised this work.

Author Information Correspondence and requests for materials should be addressed to I. B. S. (ian.spielman@nist.gov). 


\section{Methods}

SO coupled Hamiltonian Our system consisted of a $F=1$ BEC with a bias magnetic field along $\hat{y}$ at the intersection of two Raman laser beams propagating along $\hat{x}+\hat{y}$ and $-\hat{x}+\hat{y}$ with angular frequencies $\omega_{L}$ and $\omega_{L}+\Delta \omega_{L}$, respectively. The rank-1 tensor light shift of these beams produced an effective Zeeman magnetic field along the $z$ direction with Hamiltonian $\hat{H}_{R}=$ $\Omega_{R} \check{\sigma}_{3, z} \cos \left(2 k_{L} \hat{x}+\Delta \omega_{L} t\right)$, where $\check{\sigma}_{3, x, y, z}$ are the $3 \times 3$ Pauli matrices and we define $\check{1}_{3}$ as the $3 \times 3$ identity matrix. If we take $\hat{y}$ as the natural quantization axis (by expressing the Pauli matrices in a rotated basis $\check{\sigma}_{3, y} \rightarrow \check{\sigma}_{3, z}, \check{\sigma}_{3, x} \rightarrow \check{\sigma}_{3, y}$, and $\check{\sigma}_{3, z} \rightarrow \check{\sigma}_{3, x}$ ) and make the rotating wave approximation, the Hamiltonian for spin states $\left\{\left|m_{F}=+1\right\rangle,|0\rangle,|-1\rangle\right\}$ in the frame rotating at $\Delta \omega_{L}$ is

$$
\begin{aligned}
\hat{H}_{3}= & \frac{\hbar^{2} \hat{\mathbf{k}}^{2}}{2 m} \check{1}_{3}+\left(\begin{array}{ccc}
3 \delta / 2+\hbar \omega_{q} & 0 & 0 \\
0 & \delta / 2 & 0 \\
0 & 0 & -\delta / 2
\end{array}\right)+ \\
& \frac{\Omega_{R}}{2} \check{\sigma}_{3, x} \cos \left(2 k_{L} \hat{x}\right)-\frac{\Omega_{R}}{2} \check{\sigma}_{3, y} \sin \left(2 k_{L} \hat{x}\right) .
\end{aligned}
$$

As we justify below, $\left|m_{F}=+1\right\rangle$ can be neglected for large enough $\hbar \omega_{q}$, which gives the effective two-level Hamiltonian

$$
\hat{H}_{2}=\frac{\hbar^{2} \hat{\mathbf{k}}^{2}}{2 m} \check{1}+\frac{\delta}{2} \check{\sigma}_{z}+\frac{\Omega}{2} \check{\sigma}_{x} \cos \left(2 k_{L} \hat{x}\right)-\frac{\Omega}{2} \check{\sigma}_{y} \sin \left(2 k_{L} \hat{x}\right)
$$

for the pseudo-spin $|\uparrow\rangle=\left|m_{F}=0\right\rangle$ and $|\downarrow\rangle=|-1\rangle$ where $\Omega=\Omega_{R} / \sqrt{2}$. After a local pseudo-spin rotation by $\theta(\hat{x})=2 k_{L} \hat{x}$ about the pseudo-spin $\hat{z}$ axis followed by a global pseudo-spin rotation $\check{\sigma}_{z} \rightarrow \check{\sigma}_{y}, \check{\sigma}_{y} \rightarrow \check{\sigma}_{x}$, and $\check{\sigma}_{x} \rightarrow \check{\sigma}_{z}$, the $2 \times 2$ Hamiltonian takes the SO coupled form

$$
\hat{H}_{2}=\frac{\hbar^{2} \hat{\mathbf{k}}^{2}}{2 m} \check{1}+\frac{\Omega}{2} \check{\sigma}_{z}+\frac{\delta}{2} \check{\sigma}_{y}+2 \frac{\hbar^{2} k_{L} \hat{k}_{x}}{2 m} \check{\sigma}_{y}+E_{L} \check{1} .
$$


The SO term linear in $\hat{k}_{x}$ results from the non-commutation of the spatially-dependent rotation about the pseudo-spin $z$ axis and the kinetic energy.

Effective two-level system For atoms in $\left|m_{F}=-1\right\rangle$ and $\left|m_{F}=0\right\rangle$ with velocities $\hbar k_{x} / m \approx 0$ and Raman-coupled near resonance, $\delta \approx 0$, the $\left|m_{F}=+1\right\rangle$ state is detuned from resonance owing to the $\hbar \omega_{q}=3.8 E_{L}$ quadratic Zeeman shift. For $\delta / 4 E_{L} \ll 1$ and $\Omega<4 E_{L}, \Delta(\Omega, \delta) \approx \delta[1-$ $\left.\left(\Omega / 4 E_{L}\right)^{2}\right]^{1 / 2}$

Effect of the neglected state In our experiment, we focused on the two level system formed by the $\left|m_{F}=-1\right\rangle$ and $\left|m_{F}=0\right\rangle$ states. We verified the validity of this assumption by adiabatically eliminating the $\left|m_{F}=+1\right\rangle$ state from the full three level problem. To second order in $\Omega$, this procedure modifies the detuning $\delta$ and SO coupling strength $\alpha$ in Eq. 1 by

$$
\begin{aligned}
\delta^{(2)} & =\left(\frac{\Omega}{2}\right)^{2} \frac{1}{4 E_{L}+\hbar \omega_{q}} \approx \frac{1}{32} \frac{\Omega^{2}}{E_{L}} \\
\alpha^{(2)} & =\left(\frac{\Omega}{2}\right)^{2} \frac{\alpha}{\left(4 E_{L}+\hbar \omega_{q}\right)^{2}} \approx \frac{\alpha}{256}\left(\frac{\Omega}{E_{L}}\right)^{2} .
\end{aligned}
$$

In these expressions, we have retained only largest term in a $1 / \omega_{q}$ expansion. In our experiment, where $\hbar \omega_{q}=3.8 E_{L}, \delta$ is substantially changed at our largest coupling $\Omega=7 E_{L}$. To maintain the desired detuning $\delta$ in the simple 2-level model (i.e., $\Delta \approx \delta+\delta^{(2)}=0$ in Fig. 1 1 ), we changed $g \mu_{\mathrm{B}} B_{0}$ by as much as $3 E_{L}$ to compensate for $\delta^{(2)}$. We did not correct for the always small change to $\alpha$.

Although both terms are small at the $\Omega=0.2 E_{L}$ transition from miscible to immiscible, slow drifts in $B_{0}$ prompted us to locate $\Delta=0$ empirically from the equal population condition, $N_{\mathrm{T} \uparrow^{\prime}}=N_{\mathrm{T} \downarrow^{\prime}}$. As a result, $\delta$ in Eq. 1 implicitly includes the perturbative correction $\delta^{(2)}$. 
Origin of the effective interaction term The additional $c_{\uparrow \downarrow}^{\prime}$ term in the interaction Hamiltonian for dressed spins directly results from transforming into the basis of dressed spins, which are

$$
\begin{aligned}
& \left|\uparrow^{\prime}, K_{x}\right\rangle \approx\left|\uparrow, k_{x}=K_{x}+q_{\uparrow}+k_{L}\right\rangle-\epsilon\left|\downarrow, K_{x}=K_{x}+q_{\uparrow}-k_{L}\right\rangle, \text { and } \\
& \left|\downarrow^{\prime}, K_{x}\right\rangle \approx\left|\downarrow, k_{x}=K_{x}+q_{\downarrow}-k_{L}\right\rangle-\epsilon\left|\uparrow, k_{x}=K_{x}+q_{\downarrow}+k_{L}\right\rangle,
\end{aligned}
$$

where $\hbar K_{x} / m$ is the group velocity, $K_{x}=q-q_{\uparrow}$ for $\left|\uparrow^{\prime}\right\rangle$ and $K_{x}=q-q_{\downarrow}$ for $\left|\downarrow^{\prime}\right\rangle$, and $\epsilon=$ $\Omega / 8 E_{L} \ll 1$. Thus, in second quantized notation, the dressed field operators transform according to

$$
\hat{\psi}_{\uparrow}(r)=\hat{\psi}_{\uparrow^{\prime}}(r)+\epsilon e^{2 i k_{L} x} \hat{\psi}_{\downarrow^{\prime}}(r)
$$

and

$$
\hat{\psi}_{\downarrow}(r)=\hat{\psi}_{\downarrow^{\prime}}(r)+\epsilon e^{-2 i k_{L} x} \hat{\psi}_{\uparrow^{\prime}}(r),
$$

where $q_{\uparrow} \approx-\sqrt{1-4 \epsilon^{2}} k_{L} \approx-k_{L}$ and $q_{\downarrow} \approx \sqrt{1-4 \epsilon^{2}} k_{L} \approx k_{L}$. Inserting the transformed operators into

$$
\hat{H}_{\mathrm{I}}=\frac{1}{2} \int d^{3} r\left[\left(c_{0}+\frac{c_{2}}{2}\right)\left(\hat{\rho}_{\downarrow}+\hat{\rho}_{\uparrow}\right)^{2}+\frac{c_{2}}{2}\left(\hat{\rho}_{\downarrow}^{2}-\hat{\rho}_{\uparrow}^{2}\right)+c_{2} \hat{\rho}_{\downarrow} \hat{\rho}_{\uparrow}\right]
$$

gives the interaction Hamiltonian for dressed spins which can be understood order-by-order (both $c_{2} / c_{0}$ and $\epsilon$ are treated as small parameters). In this analysis, the terms proportional to $c_{2}$ are unchanged to order $c_{2} / c_{0}$, and we only need to evaluate the transformation of the spin-independent term (proportional to $c_{0}$ ). At $O(\epsilon)$ and $O\left(\epsilon^{3}\right)$ all the terms in the expansion include high spatial frequency $e^{ \pm 2 i k_{L} x}$ or $e^{ \pm 4 i k_{L} x}$ prefactors. For density distributions that vary slowly on the $\lambda / 2$ length 
scale these average to zero. The $O\left(\epsilon^{2}\right)$ term, however, has terms without these modulations, and is

$$
\hat{H}_{\mathrm{I}}^{\left(\epsilon^{2}\right)}=\frac{1}{2} \int d^{3} r\left(8 c_{0} \epsilon^{2} \hat{\psi}_{\downarrow^{\prime}}^{\dagger} \hat{\psi}_{\uparrow^{\prime}}^{\dagger} \hat{\psi}_{\downarrow^{\prime}} \hat{\psi}_{\uparrow^{\prime}}\right)
$$

giving rise to $c_{\uparrow \downarrow}^{\prime}=c_{0} \Omega^{2} /\left(8 E_{L}^{2}\right)$.

Mean field phase diagram We compute the mean-field phase diagram for a ground state BEC composed of a mixture of dressed spins in an infinite homogeneous system. This applies to our atoms in a harmonic trap in the limit of $R \gg \xi_{s}$, where $R$ is the system size, $\xi_{s}=\sqrt{\hbar^{2} / 2 m\left|c_{2}+c_{\uparrow \downarrow}^{\prime}\right| n}$ is the spin healing length and $n$ is the density. We first minimize the interaction energy $\hat{H}_{\text {I }}$ at fixed $N_{\uparrow^{\prime}, \downarrow^{\prime}}$, with an effective interaction $c_{\uparrow \downarrow}^{\prime}$ as a function of $\Omega$. The two dressed spins are either phasemixed, both fully occupying the system's volume $V$, or phase-separated with a fixed total volume constraint $V=V_{\uparrow^{\prime}}+V_{\downarrow^{\prime}}$. For the phase-separated case, minimizing the free energy gives the volumes $V_{\uparrow^{\prime}}$ and $V_{\downarrow^{\prime}}$, determined by $N_{\uparrow^{\prime}, \downarrow^{\prime}}$ and $V$. The interaction energy of a phase-mixed state is smaller than that of a phase-separated state for the miscibility condition $c_{0}+c_{2}+c_{\uparrow \downarrow}^{\prime} / 2<$ $\sqrt{c_{0}\left(c_{0}+c_{2}\right)}$, corresponding to $\Omega<\Omega_{c}$. This condition is independent of $N_{\uparrow^{\prime}, \downarrow^{\prime}}$ : for any $N_{\uparrow^{\prime}, \downarrow^{\prime}}$ the system is miscible at $\Omega<\Omega_{c}$. Then, at a given $\Omega$, we minimize the sum of the interaction energy and the single-particle energy from the Raman detuning, $\left(N_{\uparrow^{\prime}}-N_{\downarrow^{\prime}}\right) \delta / 2$, allowing $N_{\uparrow^{\prime}, \downarrow^{\prime}}$ to vary. For the miscible case $\left(\Omega<\Omega_{c}\right)$, the BEC is a mixture with fraction $N_{\downarrow^{\prime}} /\left(N_{\uparrow^{\prime}}+N_{\downarrow^{\prime}}\right) \in(0,1)$ only in the range of detuning $\delta \in\left(\delta_{0}-W_{\delta}, \delta_{0}+W_{\delta}\right)$, where $\delta_{0}=c_{2} n / 2, W_{\delta}=\left|\delta_{0}\right|\left(1-\Omega / \Omega_{c}\right)^{1 / 2}$ and $n=\left(N_{\uparrow^{\prime}}+N_{\downarrow^{\prime}}\right) / V$. For the immiscible case $\left(\Omega>\Omega_{c}\right), W_{\delta}=\left(c_{2} / 8 c_{0}\right) c_{2} n$ is negligibly small compared to $c_{2} n$.

Figure $2 \mathrm{p}$ shows the mean field phase diagram as a function of $(\Omega, \delta)$, where $\delta / E_{L}$ is dis- 
played with a quasi-logarithmic scaling, $\operatorname{sgn}\left(\delta / E_{L}\right)\left[\log _{10}\left(\left|\delta / E_{L}\right|+\left|\delta_{\min } / E_{L}\right|\right)-\log _{10}\left|\delta_{\min } / E_{L}\right|\right]$, in order to display $\delta$ within the range of interest. This scaling function smoothly evolves from logarithmic for $|\delta| \gg \delta_{\min }, \approx \operatorname{sgn}\left(\delta / E_{L}\right) \log _{10}\left|\delta / E_{L}\right|$, to linear for $|\delta| \ll \delta_{\min }, \approx \delta$, where $\delta_{\min } / E_{L}=0.001 E_{L}=1.5 \mathrm{~Hz}$.

In our measurement of the dressed spin fraction $f_{\downarrow^{\prime}}$ (see Fig. 3 z), $\delta=0$ is determined from the $N_{\mathrm{T} \uparrow^{\prime}}=N_{\mathrm{T} \downarrow^{\prime}}$ condition. We identify this condition as $\delta=\delta_{0}$ and apply it for all hold time $t_{h}$. Because $\left|\delta_{0}\right| \approx 3 \mathrm{~Hz}$ is below our $\approx 80 \mathrm{~Hz}$ RMS field noise, we are unable to distinguish $\delta_{0}$ from 0.

Recombining TOF images of dressed spins To probe the dressed spin states (Eq. 3), each of which is a spin and momentum superposition, we adiabatically mapped them into bare spins, $\left|\uparrow, k_{x}=q_{\uparrow}+k_{L}\right\rangle$ and $\left|\downarrow, k_{x}=q_{\downarrow}-k_{L}\right\rangle$, respectively. Then, in each image outside a $\approx 90 \mu \mathrm{m}$ radius disk containing the condensate for each spin distribution, we fit $n_{\mathrm{T} \uparrow^{\prime}, \mathrm{T} \downarrow^{\prime}}(x, y)$ to a gaussian modeling the thermal background and subtracted that fit from $n_{\mathrm{T \uparrow ^{ \prime }}, \mathrm{T} \downarrow^{\prime}}(x, y)$ to obtain the condensate $2 \mathrm{D}$ density $n_{\uparrow^{\prime}, \downarrow^{\prime}}(x, y)$. Thus, for each dressed spin we readily obtained the temperature, total number $N_{\mathrm{T \uparrow ^{ \prime }}, \mathrm{T \downarrow ^{ \prime }}}$, and condensate densities $n_{\uparrow^{\prime}, \downarrow^{\prime}}(x, y)$.

To analyze the miscibility from the TOF images where a Stern-Gerlach gradient separated individual spin states, we recentered the distributions to obtain $n_{\uparrow^{\prime}}(x, y)$ and $n_{\downarrow^{\prime}}(x, y)$. This took into account the displacement due to the Stern-Gerlach gradient and the nonzero velocities $\hbar k_{x} / m$ of each spin state (after the adiabatic mapping). The two origins were determined by the following: we loaded the dressed states at a desired coupling $\Omega$ but with detuning $\delta$ chosen to put all atoms 
in either $\left|\downarrow^{\prime}\right\rangle$ or $\left|\uparrow^{\prime}\right\rangle$. Since $q_{\uparrow, \downarrow}=\mp\left(1-\Omega^{2} / 32 E_{L}^{2}\right) k_{L}$ (see Fig. 11 ), these velocities $\hbar k_{x} / m=$ $\hbar\left(q_{\uparrow}+k_{L}\right) / m, \hbar\left(q_{\downarrow}-k_{L}\right) / m$ depend slightly on $\Omega$, and our technique to determine the distributions' origin accounts for this effect.

Calibration of Raman Coupling Both Raman lasers were derived from the same Ti:Sapphire laser at $\lambda \approx 804.1 \mathrm{~nm}$, and were offset from each other by a pair of AOMs driven by two phase locked frequency synthesizers near $80 \mathrm{MHz}$. We calibrated the Raman coupling strength $\Omega$ by fitting the three-level Rabi oscillations between the $m_{F}=-1,0$, and +1 states driven by the Raman coupling to the expected behavior. 

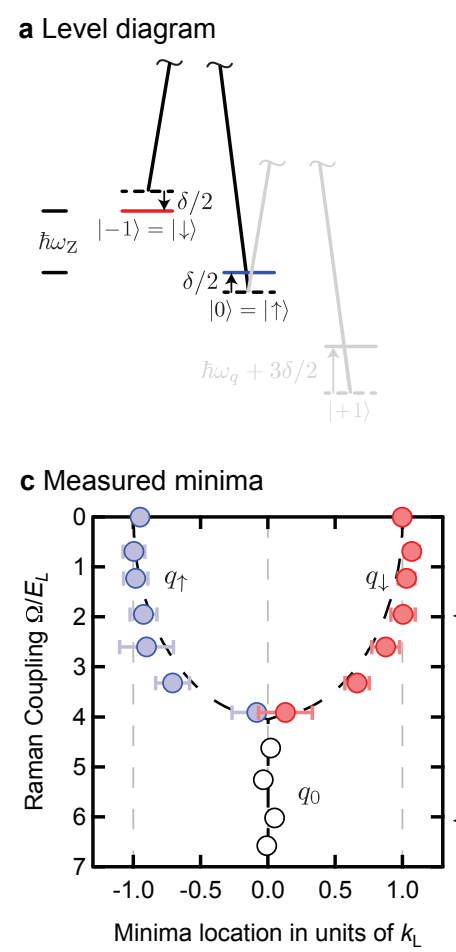

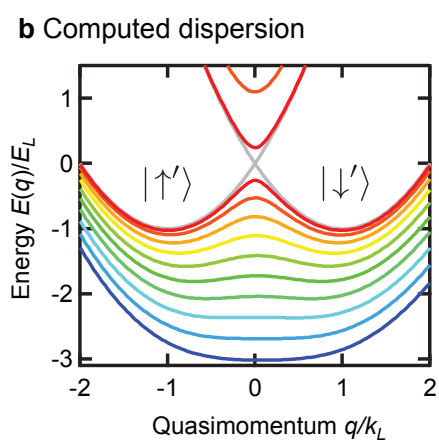

d Spin/momentum decomposition

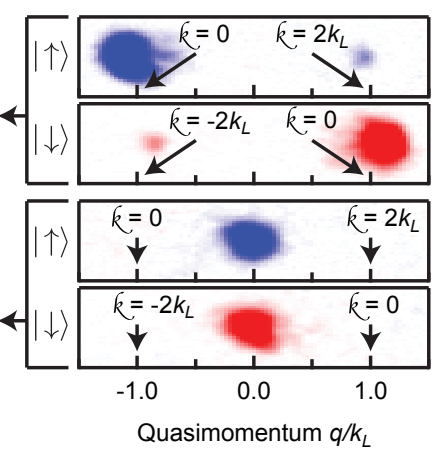

Figure 1 | Scheme for creating SO coupling. a, Two $\lambda=804.1 \mathrm{~nm}$ lasers (thick lines) coupled states $\left|F=1, m_{F}=0\right\rangle=|\uparrow\rangle$ and $\left|F=1, m_{F}=-1\right\rangle=|\downarrow\rangle$, differing in energy by a $\hbar \omega_{Z}$ Zeeman shift. The lasers, with frequency difference $\Delta \omega_{L} / 2 \pi=\left(\omega_{Z}+\delta / \hbar\right) / 2 \pi$, were detuned $\delta$ from Raman resonance. $\left|m_{F}=0\right\rangle$ and $\left|m_{F}=+1\right\rangle$ had a $\hbar\left(\omega_{Z}-\omega_{q}\right)$ energy difference; since $\hbar \omega_{q}=3.8 E_{L}$ is large, $\left|m_{F}=+1\right\rangle$ can be neglected. b. Eigenenergies at $\delta=0$ for $\Omega=0$ (grey) to $5 E_{L}$. When $\Omega<4 E_{L}$ the two minima correspond to dressed spin states $\left|\uparrow^{\prime}\right\rangle$ and $\left|\downarrow^{\prime}\right\rangle$. c, Quasimomentum $q_{\uparrow, \downarrow}$ of $\left|\uparrow^{\prime}, \downarrow^{\prime}\right\rangle$ versus $\Omega$ at $\delta=0$, corresponding to the minima of $E_{-}(q)$. Each point is averaged over about 10 experiments; the uncertainties are their standard deviation. d, Data for sudden laser turnoff: $\delta \approx 0, \Omega=2 E_{L}$ (top image-pair), and $\Omega=6 E_{L}$ (bottom pair). For $\Omega=2 E_{L},\left|\uparrow^{\prime}\right\rangle$ consists of $\left|\uparrow, k_{x} \approx 0\right\rangle$ and $\left|\downarrow, k_{x} \approx-2 k_{L}\right\rangle$, and $\left|\downarrow^{\prime}\right\rangle$ consists of $\left|\uparrow, k_{x} \approx 2 k_{L}\right\rangle$ and $\left|\downarrow, k_{x} \approx 0\right\rangle$. 

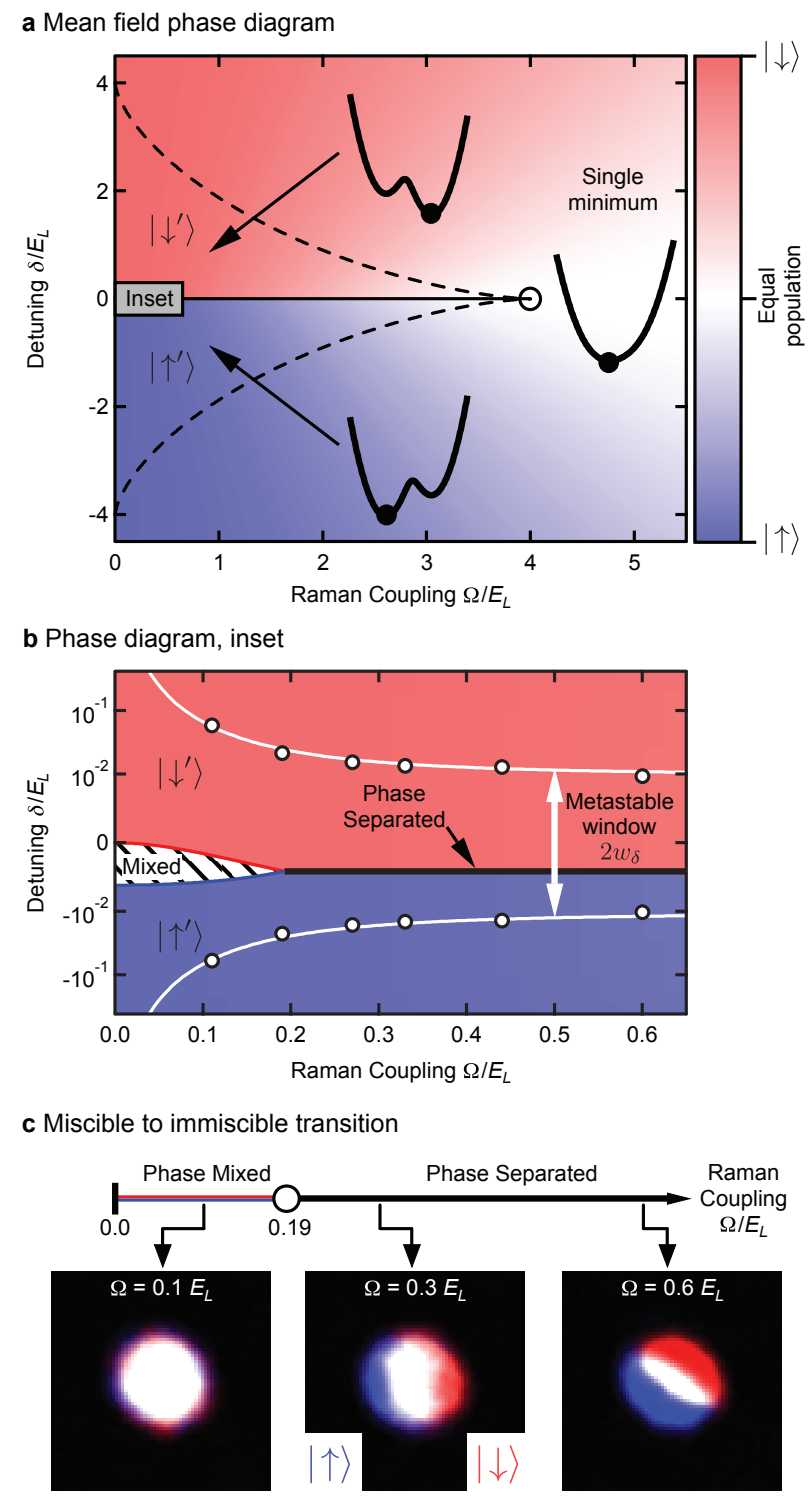

Figure 2 | Phases of a SO coupled BEC. a-b, Mean field phase diagrams for infinite homogeneous SO coupled ${ }^{87} \mathrm{Rb}$ BECs ( $1.5 \mathrm{kHz}$ chemical potential). The background colors indicate atom fraction in $|\uparrow\rangle$ and $|\downarrow\rangle$. Between the dashed lines there are two dressed spin states, $\left|\uparrow^{\prime}\right\rangle$ and $\left|\downarrow^{\prime}\right\rangle$. a, Single particle phase diagram in the $\Omega-\delta$ plane. $\mathbf{b}$, Phase diagram as modified by interactions. The dots represent a metastable region where the fraction of atoms $f_{\uparrow^{\prime}, \downarrow^{\prime}}$ remains largely unchanged for $t_{h}=3 \mathrm{~s}$. c. Phase line for mixtures of dressed spins and images after TOF (with populations $N_{\uparrow} \approx N_{\downarrow}$ ), mapped from $\left|\uparrow^{\prime}\right\rangle$ and $\left|\downarrow^{\prime}\right\rangle$ showing the transition from phase-mixed to phase-separated within the "metastable window" of detuning. 

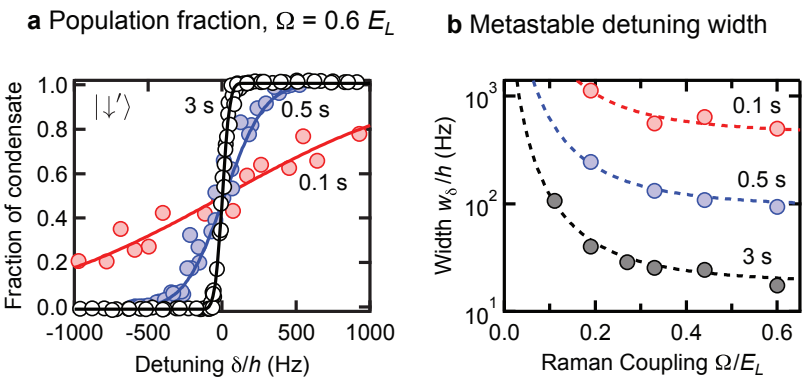

Figure 3 | Population relaxation. a, Condensate fraction $f_{\downarrow^{\prime}}$ in $\left|\downarrow^{\prime}\right\rangle$ versus detuning $\delta$ at $t_{h}=$ $0.1,0.5$, and $3 \mathrm{~s}$ showing $w_{\delta}$ decrease with increasing $t_{h}$. The solid curves are fits to the error function from which we obtained the width $w_{\delta}$. b. Width $w_{\delta}$ versus $\Omega$ at $t_{h}=0.1,0.5$, and $3 \mathrm{~s}$; the data fits well to $a\left[b+\left(\Omega / E_{L}\right)^{-2}\right]$ (dashed curves). 


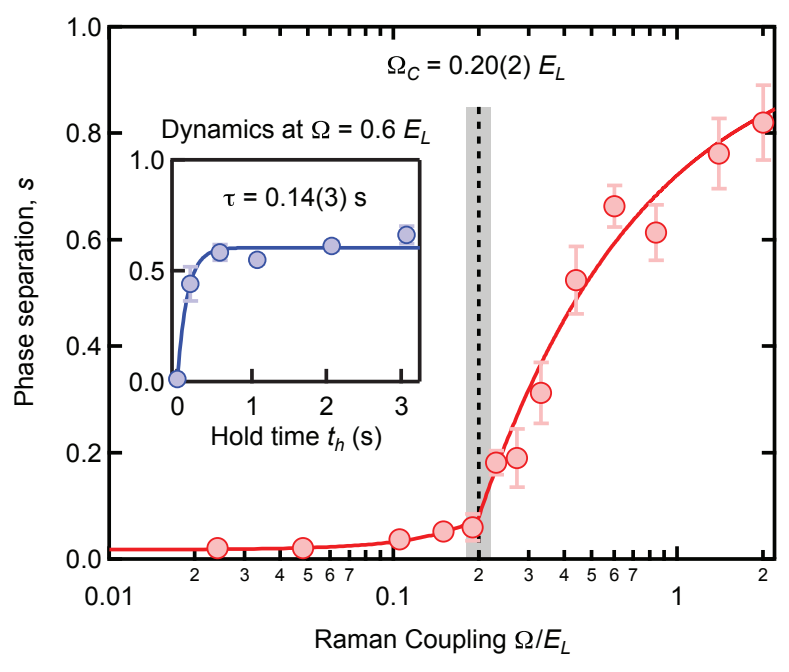

Figure 4 | Miscible to immiscible phase transition. Phase separation $s$ versus $\Omega$ with $t_{h}=3 \mathrm{~s}$; the solid curve is a fit to the function described in the text. The power law component of the fit has an exponent $a=0.75 \pm 0.07$; this is not a critical exponent, but instead results from the decreasing size of the domain wall between the regions of $\left|\uparrow^{\prime}\right\rangle$ and $\left|\downarrow^{\prime}\right\rangle$ as $\Omega$ increases. Each point represents an average over 15 to 50 realizations and the uncertainties are the standard deviation. Inset, Phase separation $s$ versus $t_{h}$ with $\Omega=0.6 E_{L}$ fit to an exponential showing the rapid $0.14(3) \mathrm{s}$ time scale for phase separation. 